\title{
Massa Anexial na Gravidez: Caso Clínico
}

\section{Adnexial Mass During Pregnancy: Case Report}

\author{
Rita Vicente Costa1* ${ }^{1 *}$ Ana Carolina Rocha ${ }^{1}$, Sofia Estevinho¹, Paula Barroso²
}

\section{RESUMO}

O diagnóstico de massas durante a gravidez é maioritariamente incidental e pouco frequente, mas tem vindo a aumentar, a par com o crescente papel da ecografia na vigilância obstétrica. Geralmente são assintomáticas, benignas, de origem ovárica e podem ter resolução espontânea.

Apresentamos o caso clínico de uma mulher de 36 anos, primigesta, grávida de 7 semanas, que recorreu ao serviço de urgência por dor hipogástrica e hemorragia vaginal, tendo sido feito o diagnóstico ecográfico de uma massa anexial multilocular sólida, com cerca de 30 cm de maior diâmetro. Às 13 semanas de gravidez foi submetida a anexectomia esquerda, por laparotomia, que decorreu sem complicações. A peça cirúrgica pesava 4955 g e o resultado histológico definitivo foi de um cistadenoma seromucinoso do ovário.

Os exames imagiológicos permitem perceber características sugestivas de benignidade ou malignidade da massa anexial, importantes no processo de decisão terapêutica. O desfecho obstétrico é geralmente favorável.

PALAVRAS-CHAVE: Doenças dos Anexos/diagnóstico por imagem; Gravidez; Quistos do Ovário/diagnóstico por imagem 


\section{ABSTRACT}

The diagnosis of masses during pregnancy is mainly incidental and infrequent but has been increasing, along with the growing role of ultrasound in obstetric surveillance. Usually the masses are asymptomatic, benign, of ovarian origin and may have spontaneous resolution.

We present the case report of a 36-year-old woman, G1P0, 7 weeks pregnant, that went to the Emergency Service due to hypogastric pain and vaginal bleeding. An adnexal complex mass with $30 \mathrm{~cm}$ (largest diameter) was diagnosed by ultrasound. At 13 weeks of pregnancy, a left adnexectomy was performed by laparotomy, with no complications. The surgical specimen weighed $4955 \mathrm{~g}$ and the final histological report was compatible with an ovarian seromucinous cistadenoma.

The imaging exams allow us to understand characteristics that suggest if an adnexal mass is benign or malignant, which is important for the therapeutic decision process. The obstetric outcome is generally favourable.

KEYWORDS: Adnexal Diseases/diagnostic imaging; Ovarian Cysts/diagnostic imaging; Pregnancy

\section{INTRODUÇÃO}

O diagnóstico de massas anexiais durante a gravidez é relativamente pouco frequente $(<10 \%)$ mas tem vindo a aumentar, a par com o crescente papel da ecografia na vigilância obstétrica. ${ }^{1,2}$ Geralmente são assintomáticas, pelo que o diagnóstico é com alguma frequência incidental e imagiológico. A maioria das massas anexiais tem origem ovárica, benigna (92\%-99\%; frequentemente cistos dermoides ou cistadenomas serosos/mucinosos) e pode ter resolução espontânea. ${ }^{2}$ A cirurgia é uma opção caso existam sintomas, potenciais complicações (nomeadamente torção, rotura, hemorragia ou infeção) ou suspeita de malignidade. ${ }^{2}$ A aparência multilocular sólida e a dimensão superior a $5 \mathrm{~cm}$ parecem ser os melhores preditores de persistência das massas. ${ }^{1}$

\section{CASO CLÍNICO}

Apresentamos o caso clínico de uma mulher de 36 anos, 10 (índice obstétrico) 0000, obesa, sem outros antecedentes pessoais de relevo, com amenorreia de 7 semanas e 4 dias e grupo sanguíneo $\mathrm{O}$ Rh+, que recorreu ao serviço de urgência por dor hipogástrica e hemorragia vaginal. Ao exame objetivo, destacava-se uma massa de consistência mole que parecia ocupar todo o abdómen. A ecografia pélvica revelou um saco gestacional in utero, embrião compatível com 7 semanas de gestação, com frequência cardíaca e ainda uma massa anexial multilocular sólida com cerca de 0,3 m de diâmetro. A utente não realizou consulta pré-concecional.

Analiticamente: b-HCG positiva (70404 UI/L), CA 125 56 U/mL, CEA, CA 15.3, CA 19.9 e parâmetros inflamatórios sem alterações.
A grávida teve alta clínica e o estudo da massa anexial foi realizado em ambulatório. A ressonância magnética mostrou uma volumosa lesão quística $(0,27 \times 0,14 \times 0,13$ m) com critérios de suspeição, origem difícil de determinar, limites bem definidos, múltiplos septos lineares e algumas vegetações na vertente posterior e inferior direita e ainda ascite em pequeno volume na cavidade pélvica e fina lâmina de ascite peri-hepática e na goteira parietocólica direita. O caso clínico foi discutido em reunião de Serviço, onde foi decidido que a terapêutica deveria ser cirúrgica e ocorrer após a ecografia obstétrica do primeiro trimestre. A referida ecografia foi realizada às 12 semanas e 4 dias de gravidez e não apresentou alterações.

Às 13 semanas de gravidez foi submetida a anexectomia esquerda, por laparotomia, que decorreu sem complicações. Fez-se colheita de líquido ascítico para estudo. A peça cirúrgica pesava 4,955 kg e o exame extemporâneo realizado foi a favor de benignidade. $O$ resultado histológico definitivo foi de um cistadenoma seromucinoso do ovário. No quarto dia de pós-operatório foi realizada uma ecografia obstétrica que revelou duas formações nodulares de 0,07 e 0,05 m, na vertente posterior do útero, sugestivas de miomas, a merecer vigilância; sem outras alterações aparentes, nomeadamente fetais. A gravidez decorreu sem intercorrências. O parto ocorreu às 39 semanas de gravidez (cesariana segmentar transversa por trabalho de parto estacionário), o recém-nascido pesava 3480 g e o seu índice de Apgar foi 9/10/10.

\section{DISCUSSÃO}

A orientação dos casos de massa anexial na gravidez não é consensual. Os exames imagiológicos permitem 
perceber características sugestivas de benignidade ou malignidade, 1 importantes no processo de decisão terapêutica. Caso se opte pela cirurgia, a escolha da via (laparotómica ou laparoscópica) depende das características da massa, da probabilidade de malignidade e da experiência da equipa médica. ${ }^{2} \mathrm{O}$ desfecho obstétrico é geralmente favorável. ${ }^{1,2}$

\section{RESPONSABILIDADES ÉTICAS}

CONFLITOS DE INTERESSE: Os autores declaram a inexistência de conflitos de interesse na realização do presente trabalho.

FONTES DE FINANCIAMENTO: Não existiram fontes externas de financiamento para a realização deste artigo.

CONFIDENCIALIDADE DOS DADOS: Os autores declaram ter seguido os protocolos da sua instituição acerca da publicação dos dados de doentes.

CONSENTIMENTO: Consentimento do doente para publicação obtido.

PROVENIÊNCIA E REVISÃO POR PARES: Não comissionado; revisão externa por pares.

\section{ETHICAL DISCLOSURES}

CONFLICTS OF INTEREST: The authors have no conflicts of interest to declare.

FINANCING SUPPORT: This work has not received any contribution, grant or scholarship.

CONFIDENTIALITY OF DATA: The authors declare that they have followed the protocols of their work center on the publication of data from patients.

PATIENT CONSENT: Consent for publication was obtained.

PROVENANCE AND PEER REVIEW: Not commissioned; externally peer reviewed.

\section{REFERÊNCIAS}

1. Nick AM, Schmeler K. Adnexal masses in pregnancy. Perinatology. 2010;1:13-19.

2. Yakasai IA, Bappa LA. Diagnosis and management of adnexal masses in pregnancy. J Surg Tech Case Rep. 2012;4:79-85. doi: 10.4103/2006-8808.110249. 